\title{
Video Article \\ Vibrodissociation of Neurons from Rodent Brain Slices to Study Synaptic Transmission and Image Presynaptic Terminals
}

\author{
Sang Beom Jun ${ }^{1,2}$, Verginia Cuzon Carlson ${ }^{1}$, Stephen Ikeda ${ }^{3}$, David Lovinger ${ }^{1}$ \\ ${ }^{1}$ Section on Synaptic Pharmacology/Laboratory for Integrative Neuroscience, National Institutes of Health/National Institute on Alcohol Abuse and Alcoholism \\ ${ }^{2}$ Department of Electronics Engineering, Ewha Womans University \\ ${ }^{3}$ Section on Transmitter Signaling/Laboratory of Molecular Physiology, National Institutes of Health/National Institute on Alcohol Abuse and Alcoholism
}

Correspondence to: David Lovinger at lovindav@mail.nih.gov

URL: https://www.jove.com/video/2752

DOI: doi:10.3791/2752

Keywords: Neuroscience, Issue 51, neuronal dissociation, synaptic transmission, GABA, calcium imaging, electrophysiology, hippocampus, striatum Date Published: 5/25/2011

Citation: Jun, S.B., Cuzon Carlson, V., Ikeda, S., Lovinger, D. Vibrodissociation of Neurons from Rodent Brain Slices to Study Synaptic Transmission and Image Presynaptic Terminals. J. Vis. Exp. (51), e2752, doi:10.3791/2752 (2011).

\section{Abstract}

Mechanical dissociation of neurons from the central nervous system has the advantage that presynaptic boutons remain attached to the isolated neuron of interest. This allows for examination of synaptic transmission under conditions where the extracellular and postsynaptic intracellular environments can be well controlled. A vibration-based technique without the use of proteases, known as vibrodissociation, is the most popular technique for mechanical isolation. A micropipette, with the tip fire-polished to the shape of a small ball, is placed into a brain slice made from a P1-P21 rodent. The micropipette is vibrated parallel to the slice surface and lowered through the slice thickness resulting in the liberation of isolated neurons. The isolated neurons are ready for study within a few minutes of vibrodissociation. This technique has advantages over the use of primary neuronal cultures, brain slices and enzymatically isolated neurons including: rapid production of viable, relatively mature neurons suitable for electrophysiological and imaging studies; superior control of the extracellular environment free from the influence of neighboring cells; suitability for well-controlled pharmacological experiments using rapid drug application and total cell superfusion; and improved spaceclamp in whole-cell recordings relative to neurons in slice or cell culture preparations. This preparation can be used to examine synaptic physiology, pharmacology, modulation and plasticity. Real-time imaging of both pre- and postsynaptic elements in the living cells and boutons is also possible using vibrodissociated neurons. Characterization of the molecular constituents of pre- and postsynaptic elements can also be achieved with immunological and imaging-based approaches.

\section{Video Link}

The video component of this article can be found at https://www.jove.com/video/2752/

\section{Protocol}

\section{Preparing Flame-Sealed Glass Micropipette}

1. Using microelectrode glass, pull a standard patch-clamp micropipette on a Flaming-Brown or equivalent micropipette puller (tip diameter $\sim 2$ $\mu \mathrm{m})$. Place micropipette tip into the flame from a Bunsen burner for $\sim 2 \mathrm{sec}$ until a fused ball forms with a diameter of 200-300 $\mu \mathrm{m}$.

2. Place flame-sealed patch pipette onto holder on a micromanipulator that can be rapidly vibrated side-to-side (travel distance $100-200 \mu \mathrm{m}$ ) using a piezoelectric bimorph, relay, or equivalently effective device.

\section{Preparing Brain Slices from P1-P21 Rats or Mice}

1. Prepare artificial cerebrospinal fluid (aCSF) with the following composition (in mM): $124 \mathrm{NaCl}, 4.5 \mathrm{KCl}, 1.2 \mathrm{NaH}_{2} \mathrm{PO}_{4}, 26 \mathrm{NaHCO}_{3}$, and $10 \mathrm{D}$ glucose. Bubble solution with $95 \%$ oxygen $/ 5 \% \mathrm{CO}_{2}$ gas for $15 \mathrm{~min}$, then add $2 \mathrm{mM} \mathrm{CaCl} 2$ and $1 \mathrm{mM} \mathrm{MgCl}$.

2. Cutting brain slices:

1. Anesthetize animal with halothane or isoflurane. Decapitate animal - remove brain - block brain in desired orientation (coronal, parasagittal, transverse, etc.) to include regions of interest.

2. Affix the blocked brain to stage of a vibrating brain slicer submerged in aCSF - section brain at 250-400 $\mu \mathrm{m}$ thickness - place slices in aCSF bubbled with carbogen in a "preincubation" chamber on netting that allows for fluid/gas exposure on all surfaces - allow slices to equilibrate in this medium for at least one hour. 


\section{Vibrodissociation}

1. Fill a $35 \mathrm{~mm}$ diameter culture dish with HEPES-buffered saline solution of the following composition (mM): $150 \mathrm{NaCl}, 5 \mathrm{KCl}, 10 \mathrm{HEPES}, 1$ $\mathrm{MgCl}_{2}, 2.5 \mathrm{CaCl}_{2}, 10 \mathrm{D}$-glucose with $\mathrm{pH}$ set to 7.4 using $\mathrm{NaOH}$ and osmolarity adjusted to $300 \mathrm{mOsM}$ using sucrose. Culture dishes may be suspension dishes, standard cell culture dishes, culture dishes with glass coverslip inserts, or dishes coated with, e.g., poly-L-lysine, depending on the need for stronger cell adherence to the dish bottom.

2. Place the slice in the culture dish and visualize with a dissecting stereoscope at $250 \mathrm{x}$. Hold slice to the bottom using a bent platinum wire ( $0.5 \mathrm{~mm}$ diameter) placed on the top surface of the slice to act as a weight.

3. Position the tip of the flame-sealed micropipette on the slice surface in the desired brain region. Activate micromanipulator to vibrate the tip laterally at $10-30 \mathrm{~Hz}$, with excursion distance $\sim 100 \mu \mathrm{m}$. Using the micromanipulator, move the tip deeper into the slice tissue such that it passes through the entire slice within $\sim 30 \mathrm{sec}$. Repeat this step as needed to maximize the number of isolated neurons obtainable from a given brain region.

4. Remove the tip from the slice - pick up the slice with forceps and gently shake it while still in solution - then remove slice completely and discard.

5. Allow the dissociated cells to settle and adhere to the dish bottom for at least $10 \mathrm{~min}$.

\section{Electrophysiological Recording}

1. Place the dish containing neurons on the stage of an inverted microscope and visualize with $10 x$ to $63 \mathrm{x}$ objective. Look for neurons with a smooth patent membrane, no blebbing, and a detectable but not oversized nucleus. If phase contrast optics are used, look for phasebright neurons with a yellowish-tinge, not too blue. Superfuse cells with extracellular solution containing desired salts, nutrients, receptor antagonists, etc. (our standard is the HEPES-buffered saline mentioned above (section 3.1)), often supplemented with $5 \mu \mathrm{M} 2,3-$ dioxo-6nitro-1,2,3,4-tetrahydrobenzo[f]quinoxaline-7-sulfonamide disodium (NBQX), and 25-100 $\mu \mathrm{M}$ D-2-amino-5-phosphonopentanoic acid (AP5) to block ionotropic glutamate receptors that allows for isolation of fast $\mathrm{GABA}_{\mathrm{A}}$ receptor-mediated IPSCs $\mathrm{s}^{1,2}$.

2. Pull a standard patch micropipette using a Flaming-Brown or equivalent puller. Pipette tip resistance should be $2-4 \mathrm{M} \Omega$ (depending on target cell size) when filled with a $\mathrm{Cl}^{-}$- based solution.

3. Establish a whole-cell recording from a visualized neuron using standard electrophysiological techniques

4. Record spontaneous postsynaptic currents (sPSCs) using a gap-free data acquisition protocol. Apply $0.2-1 \mu \mathrm{M}$ tetrodotoxin and/or low Ca ${ }^{2+}$ containing extracellular solution to record miniature postsynaptic currents (mPSCs).

5. Solutions and pharmacological agents can be directly applied to cells. We use local superfusion from fused square-tipped glass tubes with solution exchange involving lateral movement of the tubing mounted on a stepper-motor-driven micromanipulator. This allows for solution exchange in $10 \mathrm{~s}$ to $100 \mathrm{~s}$ of $\mathrm{ms}$ to achieve rapid changes in extracellular molecular content, application of receptor agonists, etc.

6. Akaike and colleagues have developed techniques to stimulate single presynaptic boutons $s^{3,4}$. A micropipette is placed near a visualized bouton and stimulation is given (negative stimulus currents of 5-10 $\mu \mathrm{A}, 0.1-0.2 \mathrm{~ms}$ duration). Unitary IPSCs are recorded, and methods such as the method of failures can be used to examine changes in presynaptic and postsynaptic function.

\section{Imaging Presynaptic Terminals}

1. Postsynaptic neurons can be filled with various dyes via the patch pipette. Neurons can also be made from mice expressing fluorescent proteins (FPs) such as green fluorescent protein (GFP) in selected cellular populations.

2. Presynaptic terminals can be visualized on vibrodissociated neurons made from mice that express FPs in particular interneuron populations. For example, mice expressing GFP driven by the glutamate decarboxylase 65 (GAD65) promoter show green somata and processes in hippocampal basket cells and other interneurons. Vibrodissociated pyramidal neurons from the hippocampal CA1 region have GFP-positive axon terminals apposed to their somata and proximal dendrites (Figure 1A). Pyramidal neurons vibrodissociated from mice that express synaptopHlourin (a pH-sensitive GFP mutant fused to the synaptic vesicle-associated VAMP2 protein) under control of the Thy1 promoter also have FP-positive attached boutons that show pH-sensitive fluorescence (Figure 1B).

3. Presynaptic boutons can be loaded with calcium indicator dyes and other fluorescent molecules using AM-esterified compounds ${ }^{5}$. Figure 2 shows an example of loading using the calcium-indicator dye Fluo4-AM. First, 1-2 $\mu \mathrm{M}$ AM-esterified dye is applied to neurons at $37^{\circ}$ for $10 \mathrm{~min}$. Dye can penetrate into the intracellular environment, where esterases cleave the molecule, yielding free, cell-impermeant and unquenched dye. This approach loads both pre- and postsynaptic cellular elements. Following the loading, the cells are washed with HEPESbuffered saline solution and kept at $37^{\circ}$ for an additional $10 \mathrm{~min}$. Prior to making a G $\Omega$-seal with a glass pipette electrode, the cells are rinsed 3 times with external buffered solution.

4. A whole-cell recording is then established using a dye-free intracellular solution. After $2-5$ min of recording, the dye is mostly removed from the postsynaptic neuron, allowing visualization of Fluo-4-loaded synaptic boutons. This technique was pioneered by Ye et al ${ }^{5}$.

5. The dye-loaded boutons can then be visualized using a high magnification, high numerical aperture objective with either a camera-based or multiphoton microscope. Simultaneous whole-cell recording and calcium imaging can be achieved using a setup as shown in Figure 3. The images of the neuron and boutons shown in Figure $2 \mathrm{C}$ were captured with an electron multiplying charge coupled device (EMCCD) camera. The power of the light source for excitation was attenuated to $1.2 \%$ with a neutral density 1.0 filter and an iris filter adjusted to $12 \%$ output. Calcium transients from the green presynaptic boutons can be observed and recorded in real time, and measured offline.

\section{Representative Results:}

\section{Spontaneous and Current Injection-Evoked Firing of Vibrodissociated Neurons}

Recordings from a typical vibrodissociated hippocampal CA1 pyramidal neuron are shown in Figure 4A. Spontaneous overshooting action potentials can be observed in pyramidal neurons dissociated from rat basolateral amygdala, hippocampus and VTA ${ }^{1,5}$. During current-clamp recordings from CA1 pyramidal neurons, hyperpolarizing current injection produces typical voltage responses while depolarizing currents 
of sufficient magnitude elicit overshooting action potentials with the typical delayed firing pattern with moderate current levels and nonaccommodating action potentials in response to stronger current injection (Figure 4B).

\section{Spontaneous and Miniature GABAergic Inhibitory Postsynaptic Currents in Vibrodissociated Neurons}

During voltage-clamp recordings with a $\mathrm{CsCl}$-based intracellular solution, spontaneous synaptic currents are observed (Figure $5 \mathrm{~A}$ ). These events

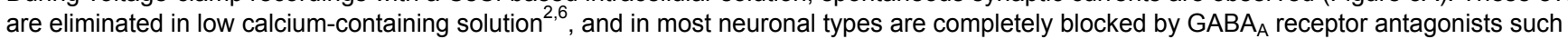
as bicuculline or gabazine (Figure 5B, C), indicating that these are sIPSCs mediated by GABA release and activation of this ionotropic receptor subtype. However, in principal neurons from brain regions such as the basolateral amygdala ${ }^{2}$ and the ventral tegmental area,7, GABA receptor $^{5,7}$ blockade reveals shorter duration EPSCs mediated by glutamatergic activation of AMPA-type receptors. Interestingly, application of TTX at concentrations that are specific for blockade of the most toxin-sensitive voltage-gated sodium channels reduces the frequency and amplitude of the sIPSCs observed in vibrodissociated neurons from several brain regions (Figure $6 A$ ) ${ }^{2,4,7}$. Thus, sodium channel activity participates in GABA release in the pinched-off synaptic boutons. It is not yet clear if full-blown sodium action potentials occur in these terminals. It is worth noting that the input resistance of a well-sealed $1 \mu \mathrm{M}$ diameter axon terminal is likely to be well into the $\mathrm{G} \Omega$ range. Thus, opening of even a small number of sodium channels might be sufficient to depolarize terminals to activate the calcium channels that mediate excitation secretion coupling. On the subject of these calcium channels, evidence suggests that $\mathrm{N}$ and $\mathrm{P} / \mathrm{Q}$-type channels participate in release at GABAergic terminals in the vibrodissociated preparations from hippocampal CA1 and elsewhere ${ }^{8}$

Modulation and plasticity of transmission by a number of neurotransmitters and receptors has been examined using vibrodissociated neurons $s^{3,4,9}$. The neurotransmitters shown to have such modulatory actions are adenosine, GABA, serotonin, endocannabinoids, etc ${ }^{2,6,10,11,12}$ In addition, short-term synaptic plasticity, such as endocannabinoid-dependent depolarization-induced suppression of inhibition (DSI) has also been described in this preparation ${ }^{2,11}$. These findings indicate that many forms of receptor-mediated and trans-synaptic signaling by endogenous neuromodulators are intact in the vibrodissociated preparation.

\section{Calcium Transients and Vesicular Release in Axon Terminals in the Vibrodissociated Preparation}

Using the EMCCD-equipped inverted microscope describe above, we have examined calcium transients in presynaptic terminals in the vibrodissociated neuron-bouton preparation. Figure 2 shows cell loading with AM-esterified Fluo-4 and subsequent postsynaptic dye dilution in a hippocampal CA1 pyramidal neuron. Spontaneous calcium transients are observed in some dye-filled boutons shown as regions of interest (ROIs) in Figure 6B. Application of $1 \mu \mathrm{M}$ tetrodotoxin (TTX) eliminates these spontaneous transients, indicating that they are mediated by activation of voltage-gated sodium currents that are spontaneously activated in boutons, leading to subsequent calcium rises. Increasing extracellular $\mathrm{KCl}$ from $10 \mathrm{mM}$ to $40 \mathrm{mM}$ with fast solution exchange produces increased fluorescence as measured in ROls that correspond to presynaptic terminals (Figure 7A). Simultaneous recording from the postsynaptic neuron is used to monitor sIPSCs and determine if event frequency is increased by high- $\mathrm{K}^{+}$depolarization (Figure 7B).

To visualize vesicle fusion in the presynaptic boutons we have used mice expressing the synaptopHlourin construct under the control of the Thy1 promoter (labeled spH21 mice). SynaptopHluorin in a molecular construct in which ecliptic pHlourin (a GFP mutant with enhanced pH sensitivity) ${ }^{12}$ is linked to the vesicle associated membrane protein (VAMP2) ${ }^{13}$. This arrangement situates the pHlourin motif in the vesicle lumen where the relatively acidic environment quenches fluorescence. Upon vesicle fusion this motif is exposed to the more neutral extracellular environment with a resultant increase in fluorescence at puncta that correspond to vesicles/presynaptic terminals. Vibrodissociation of hippocampal neurons from spH21 mice allows for visualization of fluorescent puncta of the size and location expected for GABAergic terminals (Figure 8A). Application of the high- $\mathrm{K}^{+}$- containing external solution increases fluorescence in these terminals, and this effect is blocked in the presence of an external solution in which extracellular calcium is reduced to $0.2 \mathrm{mM}$ (Figure $8 \mathrm{~B}$ ). Thus, the depolarization-induced increase in fluorescence appears to reflect excitation-secretion coupling at GABAergic synapses in the neuron-bouton preparation.

The ability to measure presynaptic calcium transients and vesicle fusion in axon terminals of the neuron-bouton preparation allows us to examine effects of neuromodulators, drugs of abuse and synaptic plasticity on presynaptic mechanisms involved in excitation-secretion coupling and exocytosis/endocytosis. These techniques can also be combined with other molecular tools and genetically-engineered mice to examine the roles of particular proteins in presynaptic function and modulation/plasticity.
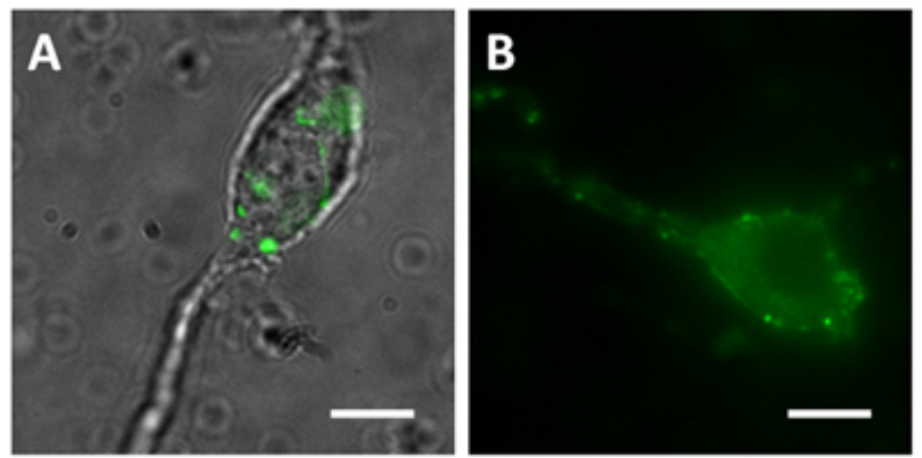

Figure 1. Vibrodissociated neurons from the hippocampal CA1 region (A) Merged image of DIC and green fluorescence images from a GAD65 mouse. DIC image is merged to clearly show the locations of the green terminals (B) Fluorescence image from a synaptophluorin (spH21) mouse. Scale bar $=10 \mu \mathrm{m}$ 

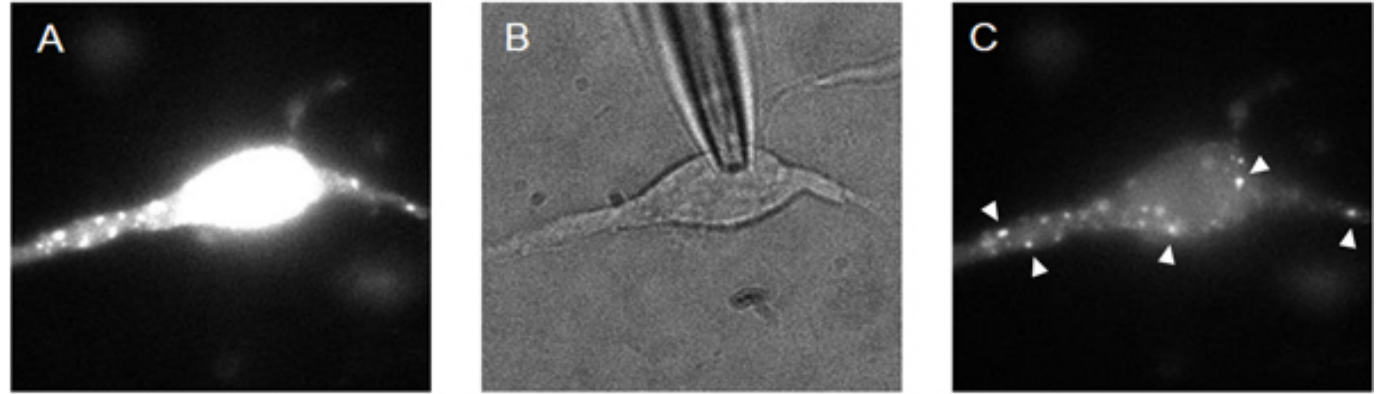

Figure 2. Calcium indicator-loading procedure: (A) whole cell is loaded with AM-esterified dye; (B) whole-cell recording dilutes dye; (C) terminals are visualized as regions of interest (arrowheads).

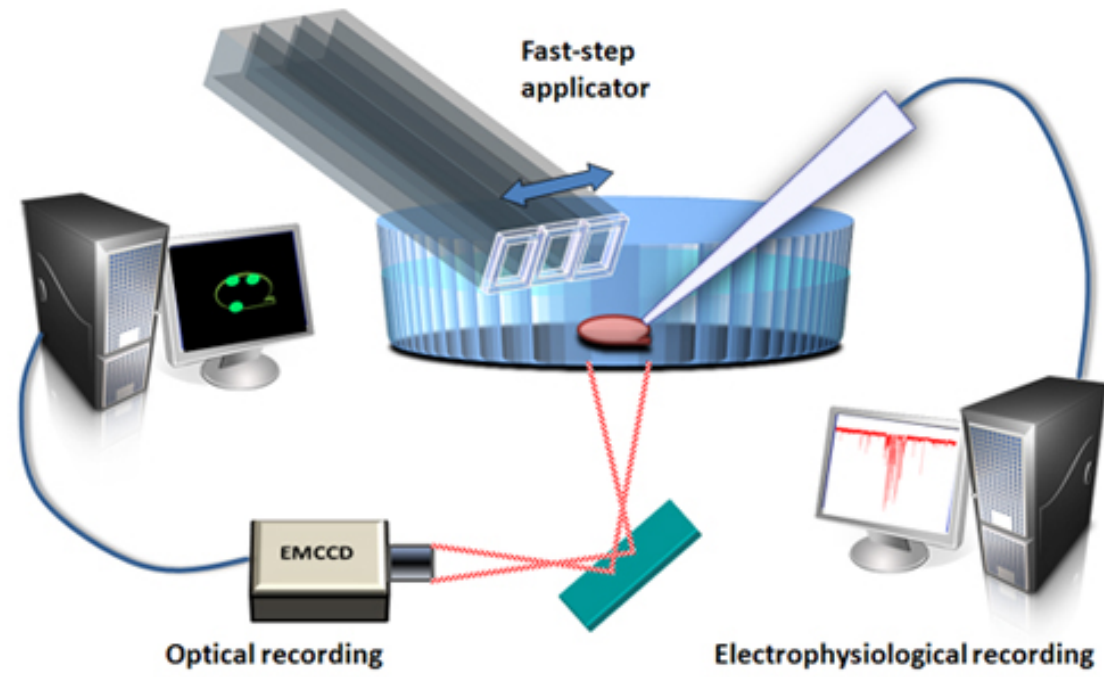

Figure 3. Schematic diagram of the experimental setup for simultaneous whole-cell recording and calcium imaging in presynaptic terminals on vibrodissociated neurons. EMCCD=electron multiplying charge coupled device.

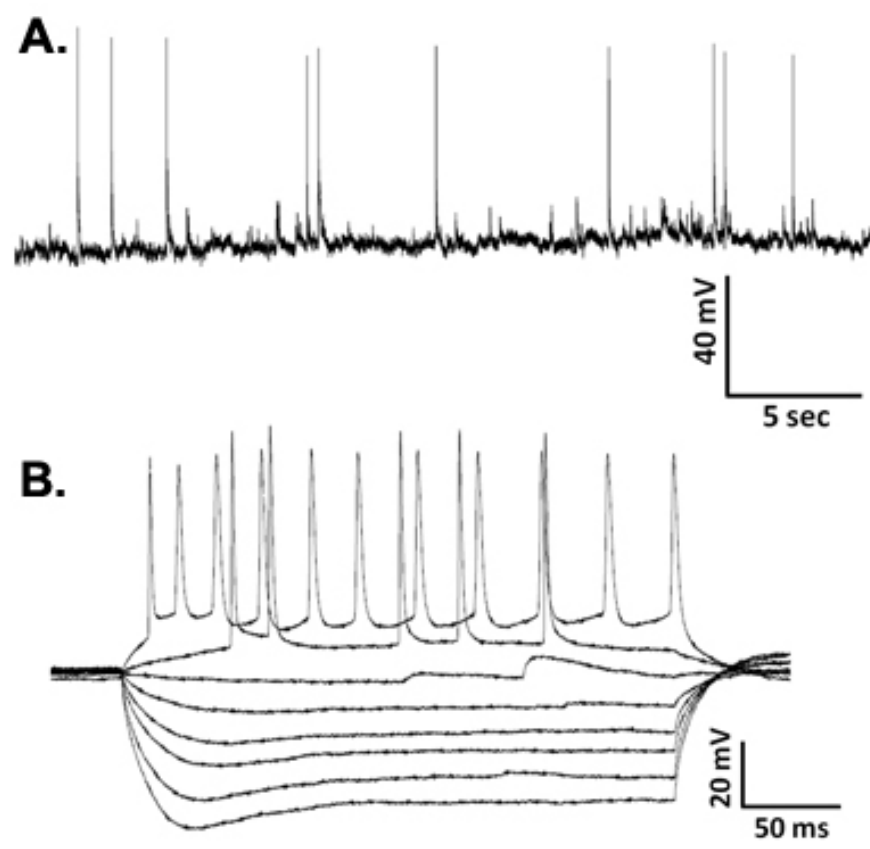

Figure 4. Representative waveforms showing (A) spontaneous action potentials from a vibrodissociated CA1 neuron and (B) membrane voltage responses to hyperpolarizing and depolarizing current injections in current-clamp recordings from a CA1 neuron. 
A.
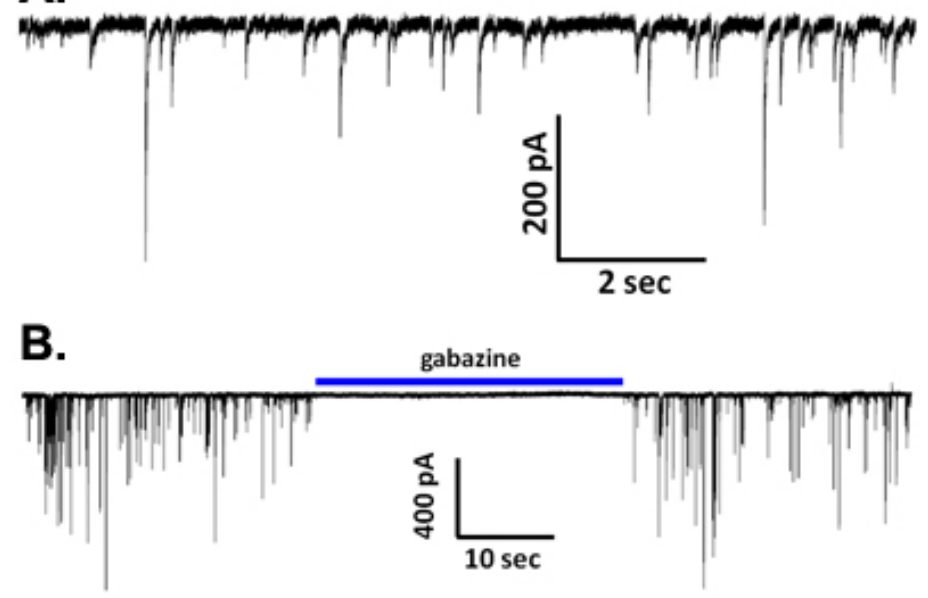

gabazine

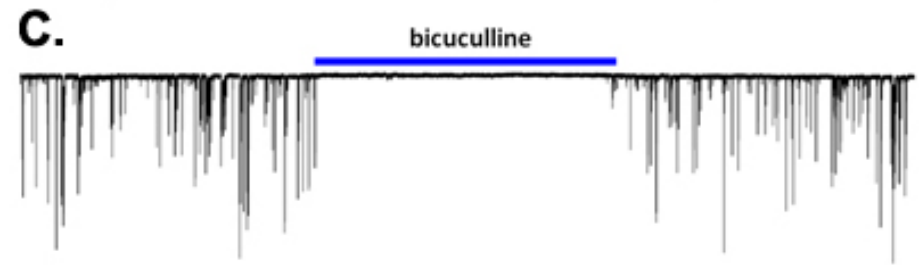

Figure 5. (A) Representative waveforms of spontaneous IPSCs from a CA1 pyramidal neuron. (B-C) The IPSCs were blocked by either gabazine $(10 \mu \mathrm{M} ; \mathrm{B})$ or bicuculline $(20 \mu \mathrm{M} ; \mathrm{C})$.

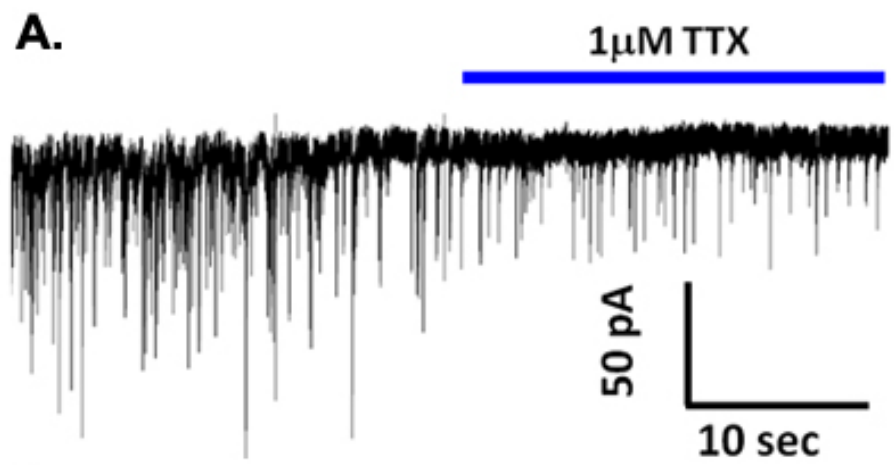

B.

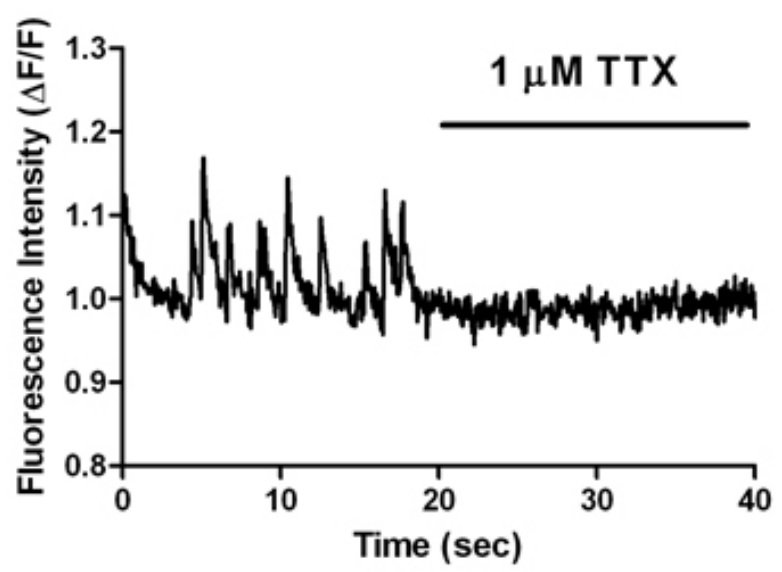

Figure 6. TTX inhibits spontaneous GABAergic synaptic transmission, and eliminates presynaptic calcium transients in the vibrodissociated hippocampal preparation. (A) Recording from a vibrodissociated neuron before and during TTX application. Note the decrease in frequency and 
amplitude of sIPSCs. (B) Calcium transients observed in a Fluo-4-loaded presynaptic terminal on a vibrodissociated neuron before and during TTX application. Note the complete loss of transients.
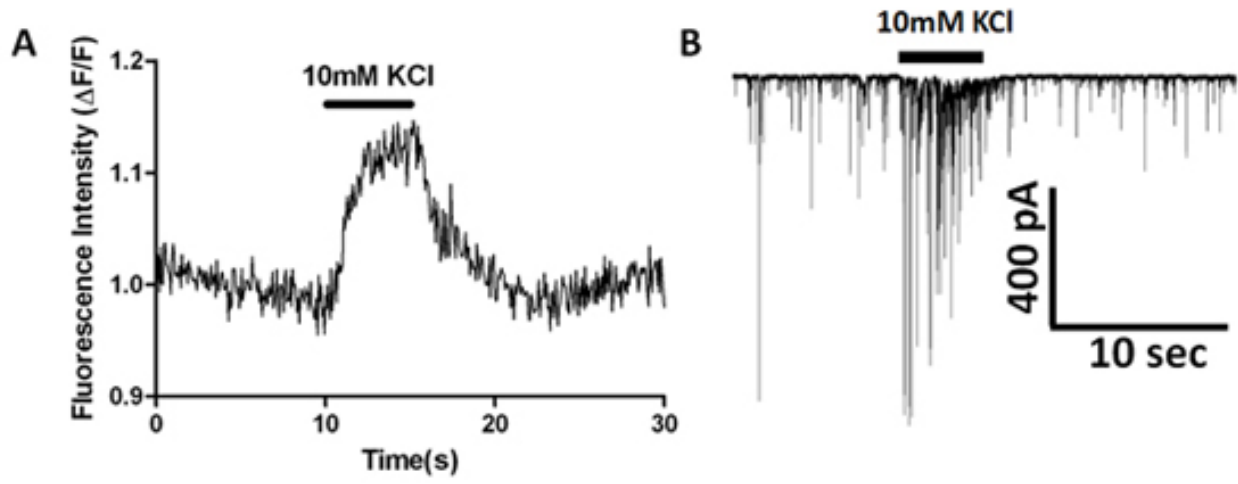

Figure 7. Simultaneous calcium indicator imaging and sIPSC whole-cell recording showing effects of high $\mathrm{K}^{+}$stimulation. (A) Fluorescence measured over time from a presynaptic bouton loaded with Fluo-4AM dye. (B) Simultaneous increase of sIPSC frequency and amplitude during high $\mathrm{K}^{+}$application.

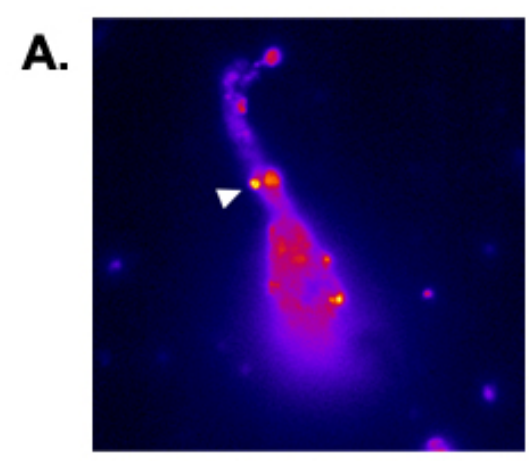

B.

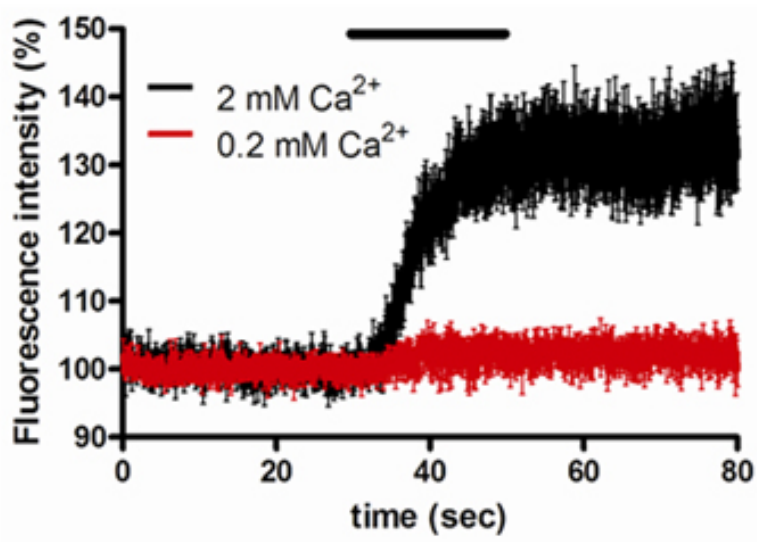

Figure 8. $\mathrm{Ca}^{2+}$-dependent high- $\mathrm{K}^{+}$response in presynaptic boutons of $\mathrm{spH} 21$ pyramidal neuron from the hippocampal CA1 region. (A) Fluorescence image of a vibrodissociated neuron. (B) High- $\mathrm{K}^{+}$application (indicated by black bar) resulted in a sustained increase in fluorescence in the presence of our normal extracellular $\mathrm{Ca}^{2+}$-containing solution $\left(2 \mathrm{mM} \mathrm{Ca} 2^{+}\right)$. No fluorescence increase was observed at low extracellular $\mathrm{Ca}^{2+}\left(0.2 \mathrm{mM} \mathrm{Ca}^{2+}\right)$. Data in graph were normalized to pre-high- $\mathrm{K}^{+}$fluorescence levels, and show average response from 3 boutons.

\section{Discussion}

Successful vibrodissociation requires that slices contain healthy neurons and that interstitial spaces are flexible enough to allow neurons to exit the slice without toxic damage. Thus, the technique works optimally at early postnatal ages (P1-21) when healthy slices can be made with less glial/interstiatial material than is found in adult brain slices. In our experience, however, optimizing slice preparation for neuronal survival in the slice itself may be counterproductive for the vibrodissociation technique. Whereas we routinely prepare slices for in situ recording using cold modified aCSF in which sucrose has been substituted for much of the extracellular $\mathrm{Na}^{+}$and $\mathrm{Ca}^{2+}$, slices prepared for vibrodissociation can be prepared (e.g. cut) in our normal recording aCSF. When preparing slices for recording from individual neurons in the slices themselves we 
also place slices in normal aCSF at $35^{\circ} \mathrm{C}$ just after sectioning and leave them for 30-60 min at this temperature before returning them to room temperature. However, slices prepared for vibrodissociation are moved immediately to room temperature just after slicing. These procedures provide a higher yield of healthy neurons after vibrodissociation, perhaps due to the lack of firm interstitial tissue that allows neurons to be more easily shaken loose from the slice itself. We have not exhaustively examined slice preparation conditions, as we routinely obtain sufficient neurons for our recording and imaging experiments on a given day. It is possible that additional modifications, such as changes in slice thickness or preincubaton procedures, could be made to increase the yield of healthy neurons. Very mild protease treatments have been tried in an effort to increase the cell yield and get the technique to work in older animals. However, invariably even mild protease treatment seems to disrupt synapse function. Thus, while the combination of protease and mechanical dissociation may still be found to work it has not proven reliable in forebrain neurons to date.

It should also be noted the relatively low yield of healthy neurons after vibrodissociation means that the technique is mainly useful for studying abundant neuronal subtypes, mainly projection neurons. The availability of GFP-expressing mice in which small neuronal subpopulations can be easily identified increases the chance of studying these rarer neurons. However, unless neuronal yield can be improved substantially accumulation of data on these neurons is likely to be relatively slow.

Several steps have proven to be crucial for successful loading of neurons with calcium-sensing dyes. Exposure to AM-esterified dye is performed at $37^{\circ} \mathrm{C}$, and we have been unsuccessful in trying to dye-load at lower temperatures. The concentration of the dyes should be optimized considering both loading time and the temperature since it appears that higher concentration of dye-loading lowers calcium concentration in the presynaptic boutons. We have observed that loading with higher concentrations decreases the frequency and the amplitude of sIPSCs. When loading calcium-sensing dyes into presynaptic terminals of vibrodissociated neurons, care must be taken because buffering capacity of the small presynaptic boutons may be different than in the soma and the sizes of boutons are not consistent. Neuron-containing dishes are washed with HEPES-buffered external solution following dye-loading, and the recovery time after washing is crucial. In addition, to make a good seal for whole-cell recording, the cells must be washed thoroughly to get rid of non-internalized dye molecules from the outer cellular membrane.

In addition to using vibrodissociated neurons for electrophysiology and live cell imaging, immunocytochemical techniques can also be applied to these cells ${ }^{11,12}$. Cells can be easily fixed and stained with a variety of antibodies, and other cell markers. The use of these cells provides a clean preparation for visualization of protein expression in GABAergic presynaptic terminals. Using mice that express fluorescent proteins under the control of promoters specific to GABAergic neurons allows the investigator to identify individual synaptic terminals in the the vibrodissociated preparation (Figure 1). Imaging fluorescent markers of this type may allow for morphological measurements in terminals using laser-based microscopy and newer techniques like STED (Stimulation Emission Depletion Microscopy). Visualization with dyes that report synaptic vesicle cycling is also possible with this preparation. Akaike and coworkers have labeled GABAergic terminals with the styryl dye, FM1-43 to visualize terminals in living cells ${ }^{4}$.

It should also be possible to perform single-cell reverse-transcriptase PCR to profile RNA expression in vibrodissociated neurons. This technique is routinely applied to enzymatically-dissociated neurons and neurons grown in cell culture.

\section{Disclosures}

No conflicts of interest declared.

\section{Acknowledgements}

We would like to acknowledge Drs. Ping Jun Zhu and Susumu Koyama for their assistance during the initial set up of the technique, and Dr. Veronica Alvarez for assistance in formatting the written manuscript. This study was funded by the Division of Intramural Clinical and Biomedical Research of NIAAA.

\section{References}

1. Zhu, P. J., \& Lovinger, D. M. Ethanol potentiates gabaergic synaptic transmission in a postsynaptic neuron/synaptic bouton preparation from basolateral amygdala. J Neurophysiol 96 (1), 433-441, (2006).

2. Zhu, P. J., \& Lovinger, D. M. Retrograde endocannabinoid signaling in a postsynaptic neuron/synaptic bouton preparation from basolateral amygdala. J Neurosci 25 (26), 6199-6207, (2005).

3. Akaike, N., \& Moorhouse, A. J. Techniques: Applications of the nerve-bouton preparation in neuropharmacology. Trends Pharmacol Sci 24 (1), 44-47, (2003).

4. Akaike, N., Murakami, N., Katsurabayashi, S., Jin, Y. H., \& Imazawa, T. Focal stimulation of single gabaergic presynaptic boutons on the rat hippocampal neuron. Neurosci Res 42 (3), 187-195, (2002).

5. Ye, J. H., Wang, F., Krnjevic, K., Wang, W., Xiong, Z. G., \& Zhang, J. Presynaptic glycine receptors on gabaergic terminals facilitate discharge of dopaminergic neurons in ventral tegmental area. J Neurosci 24 (41), 8961-8974, (2004).

6. Koyama, S., Matsumoto, N., Kubo, C., \& Akaike, N. Presynaptic 5-ht3 receptor-mediated modulation of synaptic gaba release in the mechanically dissociated rat amygdala neurons. J Physiol 529 Pt 2, 373-383, (2000).

7. Deng, C., Li, K. Y., Zhou, C., \& Ye, J. H. Ethanol enhances glutamate transmission by retrograde dopamine signaling in a postsynaptic neuron/synaptic bouton preparation from the ventral tegmental area. Neuropsychopharmacology 34 (5), 1233-1244, (2009).

8. Murakami, N., Ishibashi, H., Katsurabayashi, S., \& Akaike, N. Calcium channel subtypes on single gabaergic presynaptic terminal projecting to rat hippocampal neurons. Brain Res 951 (1), 121-129, (2002).

9. Zhu, P. J., \& Lovinger, D. M. Persistent synaptic activity produces long-lasting enhancement of endocannabinoid modulation and alters longterm synaptic plasticity. J Neurophysiol 97 (6), 4386-4389, (2007). 
10. Inada, H., Maejima, T., Nakahata, Y., Yamaguchi, J., Nabekura, J., \& Ishibashi, H. Endocannabinoids contribute to metabotropic glutamate receptor-mediated inhibition of gaba release onto hippocampal ca3 pyramidal neurons in an isolated neuron/bouton preparation. Neuroscience 165 (4), 1377-1389, (2010)

11. Sheinin, A., Talani, G., Davis, M. I., \& Lovinger, D. M. Endocannabinoid- and mglur5-dependent short-term synaptic depression in an isolated neuron/bouton preparation from the hippocampal ca1 region. J Neurophysiol 100 (2), 1041-1052, (2008)

12. Miesenbock, G., De Angelis, D. A., \& Rothman, J. E. Visualizing secretion and synaptic transmission with ph-sensitive green fluorescent proteins. Nature 394 (6689), 192-195, (1998).

13. Li, Z., Burrone, J., Tyler, W. J., Hartman, K. N., Albeanu, D. F., \& Murthy, V. N. Synaptic vesicle recycling studied in transgenic mice expressing synaptophluorin. Proc Natl Acad Sci U S A 102 (17), 6131-6136, (2005). 